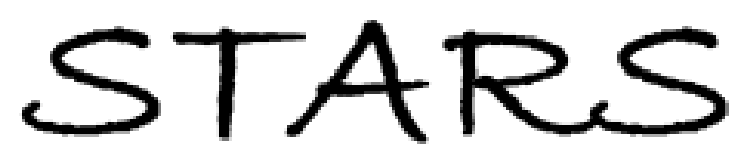

University of Central Florida

STARS

$1-1-2007$

\title{
Voigt spectral profiles in two-photon resonance fluorescence
}

Moorad Alexanian

Subir K. Bose

University of Central Florida

Find similar works at: https://stars.library.ucf.edu/facultybib2000 University of Central Florida Libraries http://library.ucf.edu

This Article is brought to you for free and open access by the Faculty Bibliography at STARS. It has been accepted for inclusion in Faculty Bibliography 2000s by an authorized administrator of STARS. For more information, please contact STARS@ucf.edu.

\section{Recommended Citation}

Alexanian, Moorad and Bose, Subir K., "Voigt spectral profiles in two-photon resonance fluorescence" (2007). Faculty Bibliography 2000s. 6816.

https://stars.library.ucf.edu/facultybib2000/6816

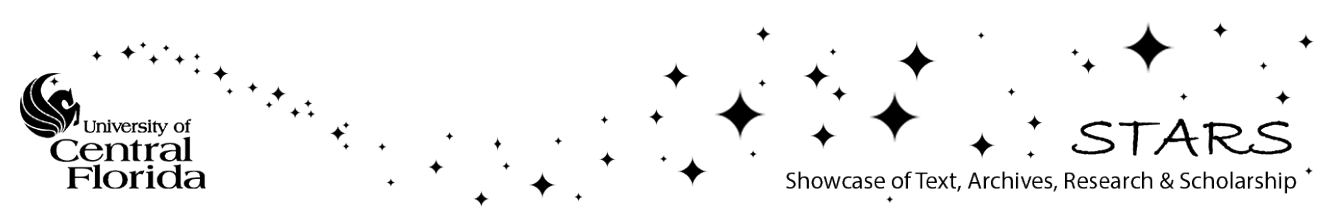




\title{
Voigt spectral profiles in two-photon resonance fluorescence
}

\author{
Moorad Alexanian* \\ Department of Physics and Physical Oceanography, University of North Carolina Wilmington, Wilmington, North Carolina 28403, USA \\ Subir K. Bose $\mathrm{B}^{\dagger}$ \\ Department of Physics, University of Central Florida, Orlando, Florida 32816, USA
}

(Received 6 June 2007; published 5 November 2007)

\begin{abstract}
A recent work on two-photon fluorescence is extended by considering the pump field to be a coherent state, which represents a laser field operating well above threshold. The dynamical conditions are investigated under which the two-photon spectrum gives rise, in addition to a Lorentzian line shape at the pump frequency, to two Voigt spectral sideband profiles. Additional conditions are found under which the Voigt profile behaves like either a Gaussian or a Lorentzian line shape.
\end{abstract}

DOI: 10.1103/PhysRevA.76.055401

PACS number(s): $42.50 . \mathrm{Hz}, 32.80 . \mathrm{Wr}$

Two-photon fluorescence has been found to have significant advantages over single-photon fluorescence in biological and medical applications [1]. Recently, we presented a theory of two-photon resonance fluorescence in which atomic excitation is by simultaneous absorption of two pump photons, followed by fluorescence emission of two fluorescent photons [2]. The atom-pump field system is treated in the "dressed atom" picture [3]. Resonance fluorescence arises from transitions among the dressed states of the atom. In this paper, we consider the case when the optical field is a coherent state, such as might be produced by an ideal laser, and show that the results shown in Figs. 1-3 of Ref. [2] for the numerical values considered actually give rise to Gaussian sidebands and not Lorentzian sidebands as incorrectly stated there.

The rate of production of the average number of fluorescent photons for arbitrary initial states of the atom and pump field is given in its most general form by Eq. (27) of Ref. [2]. This spectrum consists of four peaks: two Lorentzians at $\omega_{\mathbf{k}}=\hat{\omega}_{1}(N-2)$ and $\omega_{\mathbf{k}}=\hat{\omega}_{3}(N-2)$, and two non-Lorentzians at $\omega_{\mathbf{k}}=\hat{\omega}_{e}(N-2)$ and $\omega_{\mathbf{k}}=\hat{\omega}_{i}(N-2)$. Details are in Ref. [2]. To simplify, we consider large eigenvalues of the operator $N$ such that $N \approx N \pm j$, where $j$ is a small integer. For such cases, the rate of production of fluorescent photons becomes

$$
\begin{aligned}
\frac{1}{t}\left\langle a_{\mathbf{k} l}^{\dagger}(t) a_{\mathbf{k} l}(t)\right\rangle= & \frac{8}{\hbar^{2}}\left|\epsilon_{\mathbf{k} l}\right|^{2}\left(\left\langle\frac{\Gamma \cos ^{4} \theta}{\left[2 \omega_{\mathbf{k}}-2 \hat{\omega}_{-}(N)\right]^{2}+\Gamma^{2}} \bar{\sigma}_{33}\right\rangle\right. \\
& +\left\langle\frac{\Gamma \sin ^{4} \theta}{\left[2 \omega_{\mathbf{k}}-2 \hat{\omega}_{+}(N)\right]^{2}+\Gamma^{2}} \bar{\sigma}_{11}\right\rangle \\
& \left.+\left\langle\frac{\Gamma_{i}}{\left[2 \omega_{\mathbf{k}}-2 \omega\right]^{2}+\Gamma_{i}^{2}} \hat{m}_{i}\right\rangle\right) \\
& +\frac{4 \pi}{\hbar^{2}}\left|\epsilon_{\mathbf{k} l}\right|^{2} \delta\left(2 \omega_{\mathbf{k}}-2 \omega\right)\left\langle\hat{m}_{e}\right\rangle,
\end{aligned}
$$

where

\footnotetext{
*alexanian@uncw.edu

†skb@physics.ucf.edu
}

$$
\cos \theta=r / \sqrt{r^{2}+1}
$$

and

$$
\begin{gathered}
2 \hbar \hat{\omega}_{ \pm}(N)=2 \hbar \omega \mp \Delta / 2 \pm \frac{1}{2} \sqrt{\Delta^{2}+4 \hbar^{2}\left(g_{1}^{2}+g_{2}^{2}\right) N}, \\
\Gamma=\gamma+2 \gamma r^{2} /\left(1+r^{2}\right)^{2}, \quad \Gamma_{i}=2 \gamma\left(1+r^{4}\right) /\left(1+r^{2}\right)^{2}, \\
\hat{m}_{e}=\frac{r^{2}\left(1-r^{2}\right)}{\left(1+r^{2}\right)\left(1+r^{4}\right)}\left(\bar{\sigma}_{33}-\bar{\sigma}_{11}\right), \\
\hat{m}_{i}=\frac{2 r^{2}}{\left(1+r^{4}\right)\left(1+r^{2}\right)^{2}}\left(r^{4} \bar{\sigma}_{33}+\bar{\sigma}_{11}\right) .
\end{gathered}
$$

The spontaneous decay constant of the dressed states is given by $\gamma$, which would be the damping in a naive perturbation approach. However, the actual effective dampings of the transitions are given by $\Gamma$ and $\Gamma_{i}$. The projection operators into the $\left|\Psi_{n}^{ \pm}\right\rangle$states are $\bar{\sigma}_{11}=\sum_{n=0}^{\infty}\left|\Psi_{n}^{+}\right\rangle\left\langle\Psi_{n}^{+}\right\rangle$and $\bar{\sigma}_{33}$ $=\sum_{n=0}^{\infty}\left|\Psi_{n}^{-}\right\rangle\left\langle\Psi_{n}^{-}\right\rangle$, respectively.

Evaluation of the spectrum given by (1) requires the values of terms like $\left\langle f(N) \bar{\sigma}_{11}\right\rangle$ and $\left\langle f(N) \bar{\sigma}_{33}\right\rangle$, where $f(N)$ is a function of the operator $N$. The averaging in Eq. (1) is to be taken over the initial, disentangled atom-pump field state. Before the atoms enter the region of the pump field, the atoms could be in the ground state. Alternatively, the atoms could be prepared in a suitable superposition of the ground and the excited states. The pump field can be in a Fock state, the eigenstate $|n\rangle$ of the photon number operator with $n$ photons. Or the pump field could be a laser radiation field, which can be considered as a coherent state $|\xi\rangle$. The statistical nature of the driving field has an influence on atomic resonance fluorescence [4]

If the atom is in the ground state $|1\rangle$ and the pump field is in the Fock state $|n\rangle$, then

$$
\left\langle f(N) \bar{\sigma}_{11}\right\rangle=\cos ^{2} \theta_{n-2} f(n) \text { and }\left\langle f(N) \bar{\sigma}_{33}\right\rangle=\sin ^{2} \theta_{n-2} f(n) .
$$

Accordingly, the atom-pump field system is $|\Psi\rangle=|1\rangle$ $\otimes|n\rangle$ and so the spectrum (1) reduces to the following: 


$$
\begin{aligned}
\frac{1}{t}\left\langle a_{\mathbf{k} l}^{\dagger}(t) a_{\mathbf{k} l}(t)\right\rangle= & \frac{8}{\hbar^{2}}\left|\epsilon_{\mathbf{k} l}\right|^{2}\left(\frac{r^{4}}{\left(r^{2}+1\right)^{3}} \frac{\Gamma}{\left[2 \omega_{\mathbf{k}}-2 \hat{\omega}_{-}(n)\right]^{2}+\Gamma^{2}}\right. \\
& +\frac{r^{2}}{\left(r^{2}+1\right)^{3}} \frac{\Gamma}{\left[2 \omega_{\mathbf{k}}-2 \hat{\omega}_{+}(n)\right]^{2}+\Gamma^{2}} \\
& \left.+\frac{2 r^{4}}{\left(1+r^{4}\right)\left(1+r^{2}\right)^{2}} \frac{\Gamma_{i}}{\left[2 \omega_{\mathbf{k}}-2 \omega\right]^{2}+\Gamma_{i}^{2}}\right) \\
& +\frac{4 \pi}{\hbar^{2}}\left|\epsilon_{\mathbf{k} l}\right|^{2} \frac{r^{2}\left(1-r^{2}\right)^{2}}{\left(1+r^{4}\right)\left(1+r^{2}\right)^{2}} \delta\left(2 \omega_{\mathbf{k}}-2 \omega\right) .
\end{aligned}
$$

The inelastic spectrum consists of three Lorentzians peaks, the central peak at $\omega_{\mathbf{k}}=\omega$ and symmetric sidebands at $\omega_{\mathbf{k}}$ $=\omega_{ \pm}$, which depend on the number of pump photons $n$, i.e., the intensity of the pump field. This case has been discussed in Ref. [2] and the results of Figs. 1-3 there actually are correct for $n=200$ and not $\bar{n}=200$ as indicated there, where $\bar{n}$ is the average number of photons in the pump field.

We now analyze the case where the initial atoms are in the ground state $|1\rangle$ and the pump field is in a coherent state $|\xi\rangle$. A coherent state, a good representation of an ideal intense laser radiation, is represented by $|\xi\rangle=\sum_{n=0}^{\infty}\left(e^{-\bar{n} / 2} \xi^{n} / \sqrt{n !}\right)|n\rangle$, where $|\xi|^{2}$ is the average number $\bar{n}$ of pump photons. The initial state of the atom-radiation system is then $\sum_{n=0}^{\infty}\left(e^{-\bar{n} / 2} \xi^{n} / \sqrt{n !}\right)|1, n\rangle$. For a coherent state

$$
\left\langle f(N) \bar{\sigma}_{11}\right\rangle=\sum_{n=0}^{\infty} e^{-\bar{n}} \frac{\bar{n}^{n}}{n !} \cos ^{2} \theta_{n-2} f(n)
$$

and

$$
\left\langle f(N) \bar{\sigma}_{33}\right\rangle=\sum_{n=0}^{\infty} e^{-\bar{n}} \frac{\bar{n}^{n}}{n !} \sin ^{2} \theta_{n-2} f(n) .
$$

The rate of production of fluorescent photons is given by

$$
\begin{aligned}
\frac{1}{t}\left\langle a_{\mathbf{k} l}^{\dagger}(t) a_{\mathbf{k} l}(t)\right\rangle= & \frac{8}{\hbar^{2}}\left|\epsilon_{\mathbf{k} l}\right|^{2} \sum_{n=0}^{\infty} P_{n}\left(\frac{r^{4}}{\left(r^{2}+1\right)^{3}} \frac{\Gamma}{\left[2 \omega_{\mathbf{k}}-2 \hat{\omega}_{-}(n)\right]^{2}+\Gamma^{2}}+\frac{r^{2}}{\left(r^{2}+1\right)^{3}} \frac{\Gamma}{\left[2 \omega_{\mathbf{k}}-2 \hat{\omega}_{+}(n)\right]^{2}+\Gamma^{2}}\right. \\
& \left.+\frac{2 r^{4}}{\left(1+r^{4}\right)\left(1+r^{2}\right)^{2}} \frac{\Gamma_{i}}{\left[2 \omega_{\mathbf{k}}-2 \hat{\omega}_{i}(n)\right]^{2}+\Gamma_{i}^{2}}\right)+\frac{4 \pi}{\hbar^{2}}\left|\epsilon_{\mathbf{k} l}\right|^{2} \frac{r^{2}\left(1-r^{2}\right)^{2}}{\left(1+r^{4}\right)\left(1+r^{2}\right)^{2}} \delta\left(2 \omega_{\mathbf{k}}-2 \omega\right),
\end{aligned}
$$

where $P_{n}$, the Poisson probability distribution, is $P_{n}$ $=e^{-\bar{n}} \bar{n}^{n} / n$ !.

For large $n$, the Poisson distribution can be well approximated by a Gaussian distribution and so $P_{n}$ $\approx e^{-(n-\bar{n})^{2} / 2 \bar{n}} / \sqrt{2 \pi \bar{n}}$. The sum over the photon number states in Eq. (6) can, therefore, be replaced by an integral. These approximations allow for an analytic expression of the spectrum in terms of known functions. The first two terms of Eq. (6) give rise to a convolution of a Gaussian and a Lorentzian, i.e., the Voigt profile [5]. The third term is a pure Lorentzian centered at $\omega_{\mathbf{k}}=\omega$, resulting from the large- $n$ limit considered by us, in which case $\hat{\omega}_{i}(n) \rightarrow \omega$, which is independent of $n$. Mechanisms like Doppler and collision or pressure line broadenings give rise to the Voigt shape [6]. Numerical examples for Voigt line shapes can also be found in Ref. [6]. Therefore,

$$
\begin{aligned}
\frac{1}{t}\left\langle a_{\mathbf{k} l}^{\dagger}(t) a_{\mathbf{k} l}(t)\right\rangle= & \frac{8}{\hbar^{2}}\left|\epsilon_{\mathbf{k} l}\right|^{2}\left(\frac{r^{4}}{\left(r^{2}+1\right)^{3}} \frac{\pi \bar{\Gamma}}{\Gamma} V\left(x_{-} ; \sqrt{\bar{n}}, \bar{\Gamma}\right)\right. \\
& +\frac{r^{2}}{\left(r^{2}+1\right)^{3}} \frac{\pi \bar{\Gamma}}{\Gamma} V\left(x_{+} ; \sqrt{\bar{n}}, \bar{\Gamma}\right) \\
& \left.+\frac{2 r^{4}}{\left(1+r^{4}\right)\left(1+r^{2}\right)^{2}} \frac{\Gamma_{i}}{\left(2 \omega_{\mathbf{k}}-2 \omega\right)^{2}+\Gamma_{i}^{2}}\right) \\
& +\frac{4 \pi}{\hbar^{2}}\left|\epsilon_{\mathbf{k} l}\right|^{2} \frac{r^{2}\left(1-r^{2}\right)^{2}}{\left(1+r^{4}\right)\left(1+r^{2}\right)^{2}} \delta\left(2 \omega_{\mathbf{k}}-2 \omega\right),
\end{aligned}
$$

where

$$
\begin{aligned}
V\left(x_{ \pm} ; \sqrt{\bar{n}}, \bar{\Gamma}\right) & =\frac{1}{\pi} \int_{-\infty}^{+\infty} \frac{e^{-y^{2} / 2 \bar{n}}}{\sqrt{2 \pi \bar{n}}} \frac{\bar{\Gamma}}{\left(x_{ \pm}-y\right)^{2}+\bar{\Gamma}^{2}} d y, \\
\bar{\Gamma} & =\Gamma \frac{\sqrt{\Delta^{2}+4 \hbar^{2}\left(g_{1}^{2}+g_{2}^{2}\right) \bar{n}}}{\hbar\left(g_{1}^{2}+g_{2}^{2}\right)},
\end{aligned}
$$

and

$$
x_{ \pm}=\frac{\bar{\Gamma}}{\Gamma}\left(2 \omega_{\mathbf{k}}-2 \omega \pm \frac{\Delta}{2 \hbar} \mp \frac{\sqrt{\Delta^{2}+4 \hbar^{2}\left(g_{1}^{2}+g_{2}^{2}\right) \bar{n}}}{2 \hbar}\right) .
$$

In the integral (8), the variable of integration is $y=n-\bar{n}$ and the dominant contributions to the convolution comes from the region of $n$ near $\bar{n}$.

Note that the Voigt profile $V\left(x_{ \pm} ; \sqrt{\bar{n}}, \bar{\Gamma}\right)=\operatorname{Re}[\omega(z)] / \sqrt{2 \pi \bar{n}}$, where $w(z)=e^{-z^{2}} \operatorname{erfc}(-i z)$, erfc $z$ is the complementary error function [5], and $z=\left(x_{ \pm}+i \bar{\Gamma}\right) /(\sqrt{2} \bar{\Gamma})$.

The full width at half maximum (FWHM) of the Voigt profile is determined by the corresponding Gaussian and Lorentzian FWHM, $f_{G}$ and $f_{L}$, respectively. A reasonable approximation [7], accurate to $0.02 \%$, for the Voigt profile FWHM is given by

$$
f_{V} \approx 0.5346 f_{L}+\sqrt{0.2166 f_{L}^{2}+f_{G}^{2}}
$$

The exact expression for $f_{V}$ in terms of $f_{L}$ and $f_{G}$ has been obtained recently [8].

Therefore, the FWHM of the resonance fluorescence (7) in the variables $x_{ \pm}$is given approximately by 


$$
\Delta_{x_{ \pm}} \approx 1.0692 \bar{\Gamma}+\sqrt{0.8664 \bar{\Gamma}^{2}+8(\ln 2) \bar{n}}
$$

since $f_{L}=2 \bar{\Gamma}$ and $f_{G}=2 \sqrt{2(\ln 2) \bar{n}}$. The corresponding FWHM of the resonance fluorescence spectrum in the variable $2 \omega_{\mathbf{k}}$ is given, with the aid of (10), by

$$
\Delta_{2 \omega_{\mathbf{k}}} \approx 1.0692 \Gamma+\sqrt{0.8664 \Gamma^{2}+8(\ln 2)(\Gamma / \bar{\Gamma})^{2} \bar{n}} .
$$

Note that $\Delta_{2 \omega_{\mathbf{k}}}$ is finite, whereas $\Delta_{x_{ \pm}}$goes like $\sqrt{\bar{n}}$, for $\bar{n} \gg 1$. The asymptotic value of the ratio

$$
\frac{\bar{\Gamma}}{\sqrt{\bar{n}}}=\frac{\Gamma}{\hbar\left(g_{1}^{2}+g_{2}^{2}\right)} \frac{\sqrt{\Delta^{2}+4 \hbar^{2}\left(g_{1}^{2}+g_{2}^{2}\right) \bar{n}}}{\sqrt{\bar{n}}}
$$

determines whether the Voigt profiles in (7) reduce to a Lorentzian or Gaussian form. Therefore, from (8) one obtains that

$$
V\left(x_{ \pm} ; \sqrt{\bar{n}}, \bar{\Gamma}\right) \rightarrow \frac{1}{\pi} \frac{\bar{\Gamma}}{x_{ \pm}^{2}+\bar{\Gamma}^{2}} \quad \text { as } \frac{\bar{\Gamma}}{\sqrt{\bar{n}}} \rightarrow \infty
$$

and

[1] V. J. LaMorte, A. Zoumi, and B. J. Tromberg, J. Biomed. Opt. 8, 357 (2003); C. H. Wang, O. Y.-H. Tai, Y. Wang, T.-T. Tsai, and N.-C. Chang, J. Chem. Phys. 122, 084509 (2005); R. S. Bodaness, D. F. Heller, J. Krasinski, and D. S. King, J. Biol. Chem. 261, 12098 (1986).

[2] M. Alexanian and S. K. Bose, Phys. Rev. A 74, 063418 (2006)

[3] G. Compagno, R. Passante, and F. Persico, Atom-Field Interactions and Dressed Atoms (Cambridge University Press, Cambridge, England, 1995).

[4] P. L. Knight, W. A. Molander, and C. R. Stroud, Jr., Phys. Rev. A 17, 1547 (1978); M. G. Raymer and J. Cooper, ibid. 20,

$$
V\left(x_{ \pm} ; \sqrt{\bar{n}}, \bar{\Gamma}\right) \rightarrow \frac{1}{\sqrt{2 \pi \bar{n}}} e^{-x_{ \pm}^{2} / 2 \bar{n}} \quad \text { as } \frac{\bar{\Gamma}}{\sqrt{\bar{n}}} \rightarrow 0 .
$$

The spacing between the centers of the Lorentzians in (6) is given by $\Delta\left(2 \omega_{\mathrm{k}}\right)=2 \hat{\omega}(n+1)-2 \hat{\omega}(n)=\Gamma / \bar{\Gamma}$, while the number of levels in the width of the Gaussian is given by the standard deviation $2 \sqrt{\bar{n}}$. Accordingly, the inequality $2 \sqrt{\bar{n}}(\Gamma / \bar{\Gamma}) \ll 2 \Gamma$ corresponds to a Lorentzian shape in the Voigt spectral form, while $2 \sqrt{\bar{n}}(\Gamma / \bar{\Gamma}) \gg 2 \Gamma$ corresponds to a Gaussian. These criteria are in accordance with (15) and (16), respectively.

Therefore, for $\bar{\Gamma} / \sqrt{\bar{n}} \gg 1$, the inelastic part of the fluorescence spectrum (7) consists of three Lorentzians; the central peak at $2 \omega_{\mathbf{k}}=2 \omega$ with FWHM of $2 \Gamma_{i}$ and two sidebands at $2 \omega_{\mathbf{k}}=2 \hat{\omega}_{ \pm}(\bar{n})$ with FWHM of $2 \Gamma$, respectively. However, for $\bar{\Gamma} / \sqrt{\bar{n}} \ll 1$, the inelastic term in (7) consists of a Lorentzian central peak at $2 \omega_{\mathbf{k}}=2 \omega$ with FWHM of $2 \Gamma_{i}$ and two Gaussian sidebands at $2 \omega_{\mathbf{k}}=2 \hat{\omega}_{ \pm}(\bar{n})$ with FWHM of $(\Gamma / \bar{\Gamma}) \sqrt{8(\ln 2) \bar{n}}$, respectively. The atomic parameters used in Fig. 1 of Ref. [2], for instance, together with $\bar{n}=200$ give for the ratio $\bar{\Gamma} / \sqrt{\bar{n}} \approx 1.0 \times 10^{-3}$. Therefore, the sidebands of the spectrum for these values of the atomic parameters and $\bar{n}$ $=200$ would actually give rise to Gaussians, rather than Lorentzians, located at $2 \omega_{\mathrm{k}}-2 \omega \approx \pm 1.4 \times 10^{7} \mathrm{~s}^{-1}$ as before but with a Gaussian FWHM of approximately $1.2 \times 10^{6} \mathrm{~s}^{-1}$ rather than a Lorentzian FWHM of $2 \Gamma=3 \gamma=1.0 \times 10^{3} \mathrm{~s}^{-1}$.
2238 (1979).

[5] W. Gautschi, in Handbook of Mathematical Functions with Formulas, Graphs and Mathematical Tables, edited by M. Abramowitz and I. Stegun, Natl. Bur. Stand. Appl. Math. Ser. No. 55, 7th printing with corrections (U.S. GPO, Washington, DC, 1968), Chap. 7, Eq. (7.4.13).

[6] R. Loudon, The Quantum Theory of Light, 2nd ed. (Clarendon Press, Oxford, 1983), pp. 74-78.

[7] J. J. Olivero and R. L. Longbothum, J. Quant. Spectrosc. Radiat. Transf. 17, 233 (1977).

[8] H. O. Di Rocco, J. Quant. Spectrosc. Radiat. Transf. 92, 231 (2005). 